\title{
Dermatologie
}

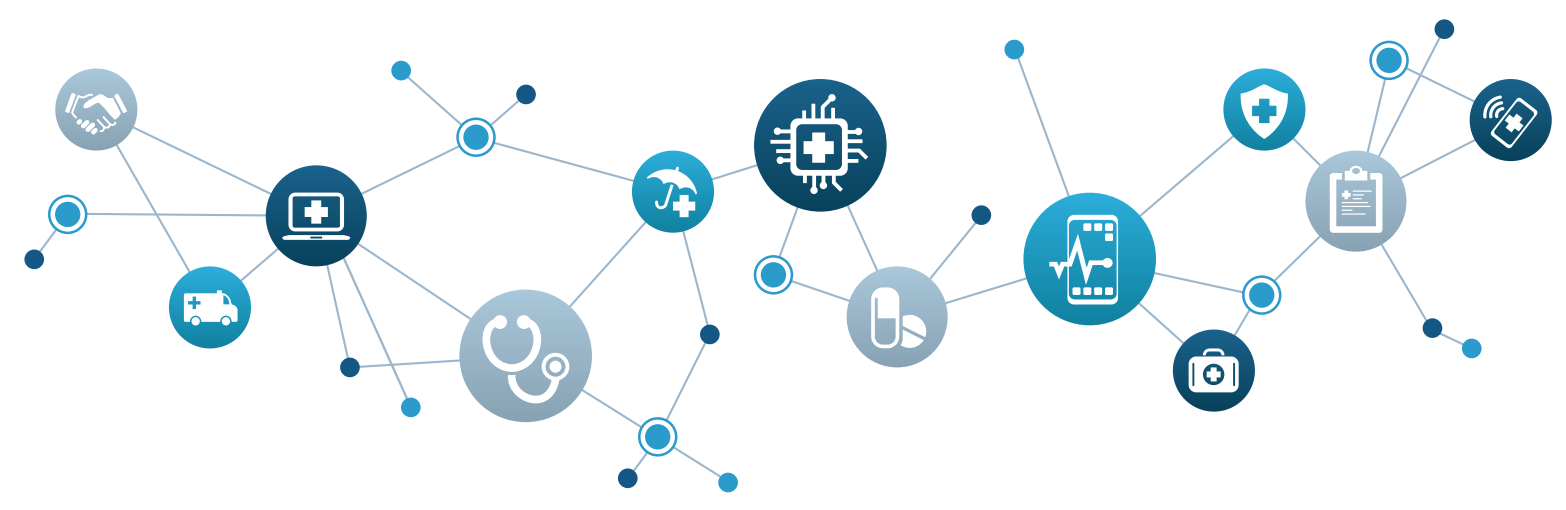

\section{Digitalisierung ist Teamarbeit}

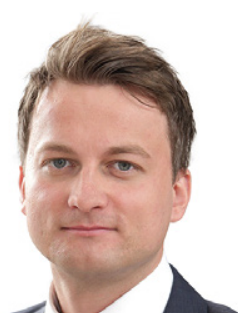

Prof. Dr. David

\section{Matusiewicz}

FOM Hochschule, KCG KompetenzCentrum für Management im Gesundheits- und Sozialwesen
Bevor wir uns weitere Gedanken zur Digitalisierung in der Dermatologie machen, sollten wir uns fragen, was Digitalisierung überhaupt ist. Ganz einfach: Digitalisierung bedeutet zunächst vor allem Konnektivität und Kommunikation. Mehr nicht.

\section{Was ist}

Die aktuelle Versorgung ist allerdings noch ziemlich analog. Patienten sind heute im Gesundheitssystem noch die, die sich anstellen müssen, um einen Termin zu bekommen. Nach einer langen Wartezeit kommen die Patienten dann - frisch gegoogelt - in die Arztpraxis, um sich dort die Zweitmeinung abzuholen. Wir haben heute schon, wenn wir ehrlich sind eine ziemliche anachronistische Präsenzkultur im Versorgungsalltag. Es stellt sich die Frage, wo morgen überwiegend behandelt wird. Die Antwort lässt sich daraus ableiten, wo wir krank werden: im Alltag. Und wo wird der Patient wieder gesund? Ebenso im Alltag. Und das geht nur digital.

\section{Was kommt}

In den USA gibt es zunehmend Ärzte, die private Hausbesuche machen, weil die Menschen sich den Arzt zuhause wünschen, der sich persönlich Zeit nimmt. Keine Parkplatzsuche und keine langen Prozesse, um in der sterilen Praxis ein paar wenige Minuten mit dem Arzt zu sprechen. Es wird die uns bisher bekannten Strukturen weiterhin geben, die Marktanteile verschieben sich nur zu immer mehr digitalen Prozessen. Die neue Formel heißt dann: mobil vor ambulant vor stationär.

Die Kommunikation wird asynchron, ubiquitär, smart und safe. Die digitale Dermatologie wird irgendwann zur Selbstverständlichkeit. Die Arztpraxis vor Ort wird etwas weniger aufgesucht und es wird mehr Zeit für den Patienten bleiben. Digital vorgeschaltete Algorithmen trennen Bagatellfälle und repetitive Tätigkeiten und die Dermatologen beschäftigen sich mit den interessanteren und komplexeren Fällen.

Wir haben durch Corona gesehen, wie wichtig die Telemedizin ist. Die Konsultation ist von wenigen Hundert in 2019 exponentiell auf über eine Million Konsultationen gestiegen. Post-Covid wird Telemedizin bzw. -dermatologie eine deutlich größere Rolle spielen als vorher. Es gibt immer mehr Menschen, die sich den «doc in my pocket» wünschen. Hier ist ein Wechsel im Mindset wichtig, damit in Zukunft eine personalisierte digitale Dermatologie funktionieren kann. Die Patienten werden aber mit ihren digitalen Füßen in Form von Wischbewegungen abstimmen.

\section{Was zählt}

Zusammenfassend geht es allerdings um das Folgende: Der Faktor Mensch bzw. die Dermatologen haben ihre Stärken und Schwäche so auch die Maschinen in Form von Apps oder Assistenzsystemen. Studien zeigen, dass medizinische Expertensysteme mittlerweile besser sind als die «menschliche» Kontrollgruppe. Bei komplexen Fällen ist der Mensch allerdings immer noch überlegen. Aber darum geht es gar nicht. Denn es ist kein Wettbewerb der Systeme. Das TEAM aus Mensch \& Maschine - also aus Dermatologie \& App - wird zunehmend als Dreamteam die jeweiligen Schwächen ausgleichen und zu einer besseren Dermatologie führen, die daten- und menschengetrieben ist. 


\section{Smart Skin, Microneedles \& Co. Von der Erweiterung unserer fünf Sinne}

Vor 50 Jahren noch schrieb man sich Briefe und benutzte Landkarten zur Orientierung. Heute tragen wir smarte Uhren und morgen lassen wir uns vielleicht schon von Kontaktlinsen navigieren [1]. Und auch in der Medizin macht sich die Digitalisierung bemerkbar [2]. PD Dr. Dr. Alexander Zink, Charlotte Steiner und Charlotte Kiani blicken in die (nahe) Zukunft.

Erst nochmal zum Anfang. Zuzeiten von Hippokrates galt in der Diagnostik der Leitsatz «Beobachtung, Befragung und Beurteilung des Patienten» - und das alles nur mit den eigenen fünf Sinnen [3]. Apparative Hilfsmittel kamen erst Jahrtausende später, so im 19. Jahrhundert das Stethoskop [2, 3]. Ende des Jahrhunderts wurden im Rahmen der Elektrifizierung mit der Entwicklung der Elektrokardiografie (EKG) und Elektroenzephalografie (EEG) weitere wichtige Meilensteine gesetzt [2]. Der Trend der zunehmenden Miniaturisierung und Mobilisierung von Elektronik zeigte sich verstärkt ab den 1950er Jahren, der Anfang war die Entwicklung von einfachen Taschenradios und später dem Walkman [4].

Im Zuge dessen wurde in den 1980er Jahren auch die Grundlage für die Entwicklung von Wearables geschaffen und - vorangetrieben durch deren großes Potenzial - zuletzt in Form der sogenannten Smart Skin weiterentwickelt. Smart Skin verspricht eine Erweiterung unserer fünf Sinne und unterstützt das ärztlich geschulte Auge bei der Behandlung von Erkrankungen, vor allem aber auch beim Monitoring von Erkrankungen sowie beim Monitoring von Gesundheit [5].

\section{Smart Skin als Chamäleon der Technik}

«Smart Skin ist in der Lage, winzige physiologische Veränderungen zu detektieren, die über lange Zeiträume nur sehr schleichend auftreten», und gleichzeitig verändert Smart Skin «die Art und Weise, wie medizinisches Personal Patienten überwachen kann» [6].

Das sind große Versprechen und wecken Neugier. Doch was genau ist Smart Skin? Gemeint ist damit ein direkt am Körper getragener
Sensor, der in Echtzeitanalyse verschiedenste Parameter messen und objektivieren kann. Die Messwerte werden dabei klassischerweise nichtinvasiv über die Haut detektiert und können unter Alltagsbedingungen vom Patienten selbst erhoben werden $[5,6]$. Gleichzusetzen mit der Farbvielfalt eines Chamäleons deckt Smart Skin ein weites Spektrum an Devices ab.

\section{Die Smartwatch war gestern,}

\section{Microneedles und epidermale Tattoos sind morgen}

Infolge des wachsenden Interesses und sich kontinuierlich weiterentwickelnder Technologien wurden bis heute schon einige Smart Skin Devices entwickelt, die sich hinsichtlich des Mechanismus sowie der Messparameter unterscheiden [6-8] und prinzipiell an unterschiedlichsten Körperregionen von Kopf bis Fuß eingesetzt werden können. Und nicht nur Vitalparameter und Schlafzyklus wie bei der klassischen Smartwatch - dem aktuell bekanntestem Vertreter der Wearables - lassen sich messen [8]. E-Textilien beispielweise lassen Rückschlüsse auf die Körpertemperatur ziehen, Kontaktlinsen können den intraokulären Augendruck messen und sogenannte Microneedles können zur Medikamentenapplikation eingesetzt werden, wie zum Beispiel in der Diabetologie [8-11]. Aktuell in Pandemiezeiten sicherlich auch von großer Relevanz sind Gesichtsmasken, die Muster in der Atemtechnik und inflammatorische Prozesse erkennen können, um Entzündungen frühzeitig zu detektieren [8, 12].

Die Smart Skin, die auch als Electronic Skin bezeichnet wird, lässt sich nach mehreren Gesichtspunkten unterteilen. So gibt es Unterschiede hinsichtlich des Materials, der Funktionsweise und auch der Technik, die ständigen Entwicklungen und Trends unterliegen $[6,10]$. In Bezug auf die Sensortechnik gibt es inzwischen etablierte elektrische und physikalische Methoden, aktuell im Trend liegen aber vor allem chemische Sensoren. Im Folgenden näher erläutert werden sollen die chemischen Sensoren nun am Beispiel eines epidermalen Tattoos zur Cortisol-Messung, entwickelt von einer Forschungsgruppe rund um Albert Salleo an der Stanford University [7].

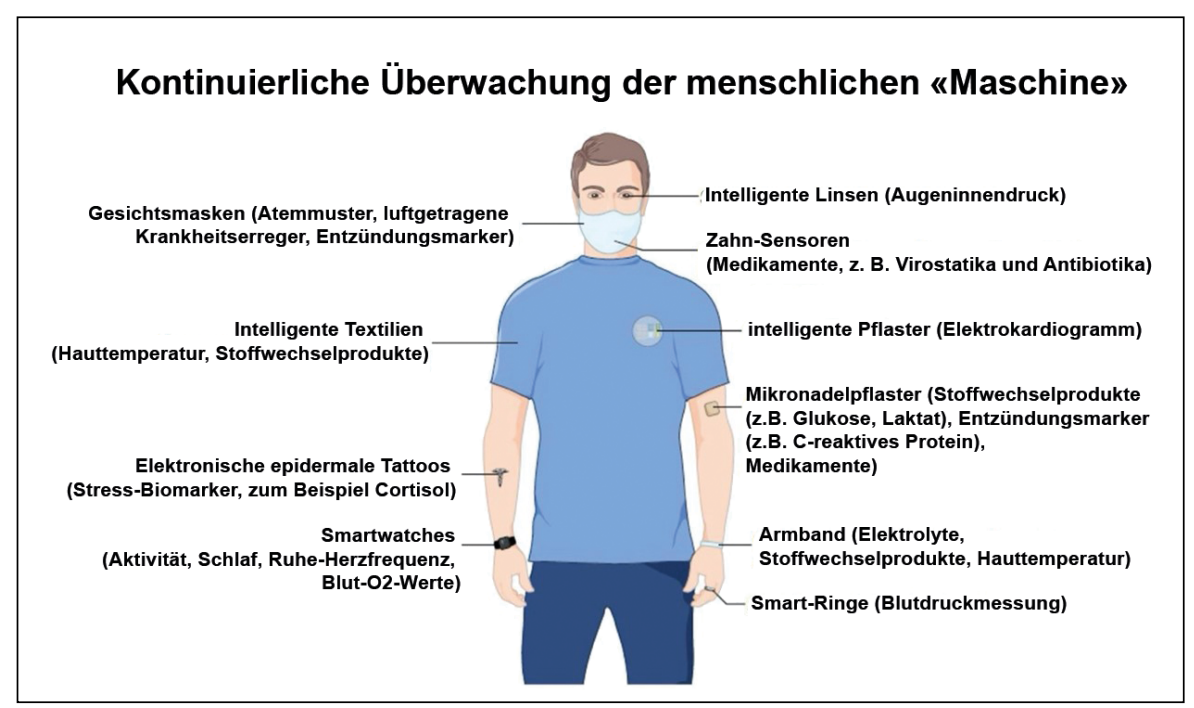

Nichtinvasive Cortisol-Messung als Tool zur Bestimmung des Stresslevels Stress wirkt sich bekanntermaßen auf vielfältige Weise auf unseren Körper aus. Unter Stressbedingungen wird das Hormon Cortisol vermehrt ausgeschüttet und kann daher als Parameter für die Bestimmung des Stresslevels dienen. Cortisol hat verschiedene physiologische Funktionen, ein konstant hoher Cortisol-Spiegel kann sich jedoch auch auf die Psyche auswirken und das Auftreten einer Depression begünstigen [13]. Deshalb kann es von klinischer Relevanz sein, den Cortisol-Spiegel sowie den Spiegelverlauf im Körper zu bestimmen. Die Forschungsgruppe um Salleo an der 
Stanford University [7] verfolgt den Ansatz, mithilfe einer nichtinvasiven Methode über Hautsensoren Cortisol im Schweiß zu messen. Über diese Methode können auch Daten von anderen Hormonen sowie von Aminosäuren, Proteinen oder Elektrolyten gesammelt werden, die wiederum Rückschlüsse auf die Stoffwechsellage des Körpers erlauben [14].

Die Messung von Cortisol im Schweiß gelang Salleo und Kollegen durch die Entwicklung einer spezifischen Membran für diesen Mechanismus. Die Membran ist in Abwesenheit von Cortisol durchlässig für lonen. Bei Anwesenheit des Stresshormons werden die Ionen jedoch geblockt und können durch den Sensor detektiert werden. Konkret bedeutet dies, dass Cortisol selbst nicht gemessen wird, sondern durch Messung der lonen Rückschlüsse auf die Cortisol-Konzentration im Schweiß gezogen werden. Gleichzeitig weisen die Messungen des Sensors jedoch eine sehr hohe Verlässlichkeit auf und werden zum Beispiel durch Körpertemperaturschwankungen nicht beeinflusst. Vielmehr wurden die Messergebnisse mit zeitgleichen Messungen durch die konventionelle ELISA-Methode (enzyme-linked immunosorbent assay) als Goldstandard eindrucksvoll verifiziert [15]. Gleichzeitig konnte bestätigt werden, dass der Sensor interessanterweise nicht auf Strukturanaloga des Cortisols, wie zum Beispiel das Cortison, reagiert und dass der Hautsensor unter normalen Alltagsbedingungen mit unterschiedlichen mechanischen Druckeinwirkungen auch messgenaue Ergebnisse liefert.

Die Entwicklung dieses Biosensors zur Bestimmung der CortisolKonzentration eröffnet der Forschung viele Möglichkeiten für die Entwicklung weiterer Sensoren, die auf dem gleichen Prinzip basieren, sich aber auf andere Hormone oder Biomoleküle beziehen [7].

\section{«Nicht App statt Arzt, sondern Arzt und App»}

Durch die Integration von Smart Skin in die ärztliche Tätigkeit kann die Diagnostik verbessert werden, Therapieeinleitungen können beschleunigt und Krankheitsverläufe besser kontrolliert werden. Mit dem Tragen einer Smart Skin könnten langfristig und regelmäBiger Werte gesammelt werden, die ein Monitoring der Patienten in ihrer täglichen komfortablen Umgebung ermöglichen. So kann zum Beispiel differenziert werden zwischen Werten, die während eines Arztbesuches gemessen wurden, oder solchen von zu Hause in «Ruhe». Das Ziel dabei ist, Störeffekte wie Nervosität zu erkennen und herauszufiltern, was medizinischen Leistungserbringern eine präzisere Beurteilung von Gesundheit und Krankheit bereits in sehr frühen Stadien erlauben würde.

Das Potenzial der Wearables erscheint in diesem Kontext enorm und noch lange nicht ausgeschöpft. Vor allem die Integration in den Alltag von Patienten bietet viel Spielraum für neue Entwicklungen, wie zum Beispiel durch die Ergänzung mit Mobile Health (mHealth). Durch mHealth kann mit dem Einsatz von digitalen Assistenten eine Schnittstelle zwischen Wearables, Patienten und Ärzten geschaffen werden. Die von Parmanto et al. [16] entwickelte SkinCare App beinhaltet genau dies: Selbstpflegeaufgaben, die Überwachung des Hautzustands und die sichere Zwei-Wege-Kommunikation zwischen Patienten und Ärzten. Das System kann den Patienten so in seiner Therapie besser unterstützen und gleichzeitig die Kommunikation zwischen Patienten und Ärzten erleichtern. Dabei sollte man mHealth und Wearables keinesfalls als Gegner oder Konkurrenz des Arztes ansehen, sondern als Freund und Unterstützer nach dem Leitspruch des Gesundheitsministers: «Nicht App statt Arzt, sondern Arzt und App» [17].

\section{Literatur}

1. Shetty S, Panait L, Baranoski J, et al.: J Surg Res 2012;177:191-195.

2. Bartmann F: Internist 2019;60:319-323.

3. Diepgen P: Geschichte der Medizin, vol 1, ed 2. Chicago, G.J. Göschen, 1913.

4. Condeco Software: The History of Wearable Technology. https://www.condecosoftware.com/blog/the-history-of-wearable-technology/, 2018 (Zugriff am 16.05.2021).

5. Hammock ML, Chortos A, Tee BC, et al.: Adv Mater 2013;25:5997-6038.

6. Someya T, Amagai M: Nat Biotechnol 2019;37:382-388.

7. Parlak O, Keene ST, Marais A, Curto VF, Salleo A: Sci Adv 2018;4:eaar2904.

8. Ates HC, Yetisen AK, Güder F, et al.: Nat Electron 2021;4:13-14.

9. Ismar E, Kurşun Bahadir S, Kalaoglu F, et al.: Glob Chall 2020;4:1900092.

10. Kim J, Kim M, Lee MS, et al.: Nat Commun 2017;8:14997.

11. Chen G, Yu J, Gu Z: J Diabetes Sci Technol 2019;13:41-48.

12. Maier D, Laubender E, Basavanna A, et al.: ACS Sensors 2019;4:2945-2951.

13. Qin DD, Rizak J, Feng XL, et al.: Sci Rep 2016;6:30187.

14. Emaminejad S, Gao W, Wu E, et al.: Proc Natl Acad Sci U S A 2017;114:4625-4630.

15. Jang HJ, Lee T, Song J, et al.: ACS Appl Mater Interfaces 2018;10:16233-16237.

16. Parmanto B, Pramana G, Yu DX, Fairman AD, Dicianno BE: BMC Med Inform Decis Mak 2015;15:114.

17. Bundesgesundheitsministerium, Spahn J: https://www.bundesgesundheitsministerium. de/presse/interviews/interviews/handelsblatt-110719.html, 2019 (Zugriff 16.05.2021).

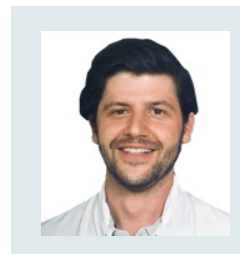

Charlotte Steiner*, Charlotte Kiani*, Alexander Zink (im Bild); Klinik und Poliklinik für Dermatologie und Allergologie am Biederstein, Fakultät für Medizin, Technische Universität München, München, Deutschland ( ${ }^{*}$ Charlotte Steiner und Charlotte Kiani haben gleichermaßen zu diesem Artikel beigetragen.) Kontaktadresse: Priv.-Doz. Dr. Dr. med. Alexander Zink, alexander.zink@tum.de 


\section{Leitlinie «Teledermatologie» Orientierung und Empfehlungen für die Praxis}

Seit Oktober 2020 gibt es sie: Die S2k-Leitlinie Teledermatologie. Darin zu finden sind Empfehlungen, wie in der Telemedizin mit verschiedenen dermatologischen Erkrankungen verfahren werden sollte, darunter Psoriasis, atopische Dermatitis und Hautkrebs. Dr. Natalia Kirsten von der Uniklinik Hamburg-Eppendorf war Teil der Expertenkommission und fast für uns die wesentlichen Punkte der Leitlinie zusammen.

Die Einführung telemedizinischer Anwendungen in der dermatologischen Versorgung hat in den letzten Jahren exponentiell zugenommen. Die Nutzung dieser bedarf jedoch nicht nur seitens der Anbieter einer Festlegung von Standards, sondern muss auch für die ärztliche Versorgung klar definiert werden.

Die Telemedizin stellt eine zeitgemäße und sinnvolle Ergänzung zur klassischen Vor-Ort-Versorgung dar. Um eine sachgerechte Anwendung zu gewährleisten und eine Grundlage für die Festsetzung gesetzlicher Rahmen in der Beurteilung telemedizinischer Versorgungskonzepte festzulegen, wurde die Leitlinie «Teledermatologie» verfasst. Die Fachgruppe der Dermatologen ist die erste, die die Standards der Telemedizin in einer Leitlinie gebietsbezogen festgelegt hat.

\section{Definitionssache}

Einleitend beschäftigt sich die Leitlinie mit den Begriffsklärungen. Die taxonomische Darstellung der Telemedizin bindet diese als einen Teilbereich in den Gesamtkontext von eHealth ein. Die Begriffe «eHealth» und «Digital Health» werden gleichgesetzt. Telemedizin wird in der Leitlinie als Fernübertragung und -nutzung digitaler Daten definiert. Dies impliziert auch die Nutzung von Apps und anderer ergänzender Anwendungen neben den klassischen Formen der Telemedizin.

Die Leitlinie hatte zum Ziel, die Evidenz der teledermatologischen Anwendungen zusammenzufassen und so Empfehlungen für die klinische Praxis zu generieren. Aufgrund der Vielzahl von Apps und zum Teil noch fehlender einheitlicher Evaluation und Evidenz befasst sich die Leitlinie ausschließlich mit der klassischen telemedizinischen Versorgung unter Ausschluss der Apps. Ergänzend zur Leitlinie sei hierfür auf den Leitfaden zur Telemedizin hingewiesen, der kürzlich aktualisiert wurde.

In der Leitlinie werden 2 Arten der telemedizinischen Versorgung adressiert: die Real-Time (RT)-Methodik und die Store-and-Forward (SAF)-Methodik. RT bezeichnet telemedizinische Versorgung in Echtzeit und SAF erlaubt eine asynchrone Übertragung von Daten vom Sender (z.B. Patient/in) an den Empfänger (z.B. Arzt/Ärztin) mit dem einen Zwischenschritt der Speicherung von fotografischen Befunden und anamnestischen Angaben auf einem externen Server. Der Empfänger hat dabei die Möglichkeit, die Anfrage asynchron zu bearbeiten und die Antwort an den Sender zeitversetzt zu verschicken.

\section{Empfehlungen für die Praxis}

Aufgrund der aktuellen Evidenzlage werden in der Leitlinie 4 Diagnosegruppen einzeln adressiert und zum Schluss allgemeine Empfehlungen für andere Anwendungsbereiche ausgesprochen. Bei den einzeln adressierten Diagnosen handelt es sich um: Psoriasis, Neurodermitis und andere Ekzemerkrankungen, Hautkrebs und chronische und akute Wunden.

\section{Psoriasis und Ekzemerkrankungen}

Die Empfehlungen für die beiden Diagnosegruppen sind sehr ähnlich und werden deshalb hier zusammengefasst. Für diese beiden chronisch-entzündlichen Diagnoseklassen gibt es randomisierte kontrollierte Studien, die den Nutzen telemedizinscher Verfahren untersucht und hinreichend bewiesen haben.

Laut Leitlinie soll die Erstdiagnostik der Psoriasis oder der Ekzemerkrankungen nicht rein telemedizinisch erfolgen. Besonders auf die Erhebung eines ganzheitlichen Befundes unter Berücksichtigung von Komorbiditäten und deren Ausprägung sei hier verwiesen, so zum Beispiel der Hinweis auf das Vorliegen einer Psoriasis-Arthritis bei Patienten mit einer bestehenden Psoriasis vulgaris. Eine offene Empfehlung besteht für eine rein telemedizinische Verlaufsbeurteilung für die Psoriasis sowie die Ekzemerkrankungen nach einer in Präsenzuntersuchung erfolgten Diagnosestellung. Die Empfehlung gilt für die RT- wie auch für die SAFMethodik. Beide Diagnoseklassen erfordern im Verlauf die Beurteilung der Morphologie sowie der Lebensqualität für eine optimale Versorgung. Dies kann laut Leitlinie telemedizinische erfolgen, wenngleich die Erhebung der gängigen Scores, wie dem PASI (Psoriasis Area and Severity Index) oder EASI (Eczema Area and Severity Index), für die Beurteilung des Schweregrades der Erkrankung gewisse Limitationen zeigt. So kann für die Bestimmung des PASI die Variable «Induration» und beim EASI die Variable «Ödem» telemedizinisch nicht hinreichend beurteilt werden. Hier wird zukünftig der Einsatz von validierten Messinstrumenten für die Erhebung des Schweregrades durch den Patienten selbst an Gewicht gewinnen, wie zum Beispiel dem SAPASI (Self-administered PASI).

\section{Hautkrebs}

Im Bereich der teledermatologischen Forschung gibt es für die melanozytären und nicht melanozytären Läsionen die beste qualitative Evidenz sowohl für die mobile als auch für die webbasierten Lösungen. Bei einer Überlegenheit der Präsenzbeurteilung in einzelnen Studien soll der reine Einsatz telemedizinischer Lösungen für die Diagnosestellung laut Leitlinie fallbasiert und individuell erwogen werden.

Im Gegensatz zur Psoriasis oder den Ekzemerkrankungen kann die Primärdiagnostik melanozytärer und nicht melanozytärer Läsionen rein telemedizinisch erfolgen. Ist der Befund nicht eindeutig, soll eine dermatoskopische Beurteilung erfolgen. Diese kann telemedizinsch oder aber in Präsenz stattfinden. Bei jedem Befund soll si- 
Tab 1.: Zusammengefasste Empfehlungen der S2k-Leitlinie «Teledermatologie»

\begin{tabular}{|c|c|c|c|}
\hline Diagnose & $\begin{array}{l}\text { Primär- } \\
\text { diagnostik }\end{array}$ & $\begin{array}{l}\text { Verlaufs- } \\
\text { beobachtung }\end{array}$ & Besonderheiten \\
\hline Psoriasis & - & + & Erhebung des Schweregrades mit dem SAPASI \\
\hline $\begin{array}{l}\text { Neurodermitis und andere Ek- } \\
\text { zemerkrankungen }\end{array}$ & - & + & $\begin{array}{l}\text { Die Berechnung des EASI-Wertes kann nicht erfolgen, da die Variable } \\
\text { «Ödem» telemedizinisch nicht hinreichend beurteilt werden kann. }\end{array}$ \\
\hline $\begin{array}{l}\text { Melanozytäre und nicht mela- } \\
\text { nozytäre Hautläsionen }\end{array}$ & + & + & $\begin{array}{l}\text { Die Diagnosestellung sollte nicht allein auf KI-basierten Lösungen } \\
\text { beruhen. }\end{array}$ \\
\hline Chronische und akute Wunden & + & + & $\begin{array}{l}\text { Die supportive telemedizinische Beurteilung ist einer Standardver- } \\
\text { sorgung zum Teil überlegen; zusätzlicher edukativer Effekt beim Pfle- } \\
\text { gepersonal. }\end{array}$ \\
\hline $\begin{array}{l}\text { Andere dermatologische An- } \\
\text { wendungsgebiete }\end{array}$ & $(+)$ & + & $\begin{array}{l}\text { Für einzelne Indikationen besteht noch unzureichende Evidenz für } \\
\text { den Nutzen telemedizinischer Verfahren. }\end{array}$ \\
\hline \multicolumn{4}{|c|}{ SAF-Anwendungen gleichwertig mit den RT-Anwendungen in der Qualität der Diagnosestellung. } \\
\hline \multicolumn{4}{|c|}{$\begin{array}{l}\text { «-» Reine telemedizinische Versorgung nicht empfohlen. } \\
\text { «+» Reine telemedizinische Versorgung empfohlen. } \\
\text { «(+)» Reine telemedizinische Versorgung bei klaren morphologischen Befunden empfohlen. }\end{array}$} \\
\hline
\end{tabular}

chergestellt sein, dass alle notwendigen anamnestischen sowie klinischen Informationen vorliegen, die die Diagnosestellung ergänzend beeinflussen könnten.

Für die Beurteilung melanozytärer Läsionen wurden schon mehrere, auf Künstlicher Intelligenz (KI) basierende Lösungen entwickelt. Diese können supportiv eingesetzt werden. Von einer rein $\mathrm{KI}$-basierten Diagnosestellung soll laut Leitlinie abgesehen werden.

\section{Chronische und akute Wunden}

Es besteht eine gute Evidenz für den Nutzen telemedizinscher Verfahren in der Beurteilung und Versorgung chronischer und akuter Wunden. Da ein zusätzlicher Nutzen für eine ergänzende telemedizinische Verlaufsbeurteilung in der Versorgung von Patienten mit chronischen Wunden gezeigt werden konnte, ist der Einsatz telemedizinischer Verfahren besonders in diesem Indikationsfeld sinnvoll. Laut Leitlinie kann sowohl die Primärdiagnostik als auch die Verlaufsbegutachtung telemedizinisch erfolgen und sinnvoll sein. Es ist sicherzustellen, dass alle für die valide Diagnosestellung notwendigen Befunde und anamnestischen Angaben vorliegen.

Ein zusätzlicher positiver Effekt wird der telemedizinisch begleitenden Edukation von Pflegepersonal zugesprochen, zum Beispiel beim Anlegen von Kompressionsverbänden.

\section{Weitere dermatologische Anwendungsbereiche}

Abschließend befasst sich die Leitlinie mit weiteren Anwendungsbereichen in der Dermatologie. Grundsätzlich besteht die Empfehlung zur telemedizinischen Verlaufsbeobachtung von Bestandspatienten. Da grundsätzlich kein Vorteil der RT-im Vergleich zur SAF-Methodik besteht, wird hier vor allem die SAF-Methodik empfohlen. Die SAF-Versorgung kann dabei in der Primärdiagnostik von morphologisch eindeutigen Dermatosen eigesetzt wer- den. Auch hier ist sicherzustellen, dass die Vollständigkeit der diagnostischen Maßnahmen und der anamnestischen Angaben vorliegt oder im Zuge der telemedizinischen Behandlung initiiert wird.

\section{Zusammenfassung}

Die S2k-Leitlinie Teledermatologie ist die erste ihrer Art, die gebietsbezogene Empfehlungen für die telemedizinische Versorgung von Patienten gestellt hat.

Die Verlaufsbeobachtung kann für viele Indikationen, insbesondere bei bekannten Patienten empfohlen werden. Es liegt jedoch in der Verantwortung des behandelnden Arztes, die Diagnosestellung primär rein telemedizinisch durchzuführen. Dabei ist auf die Vollständigkeit der anamnestischen Angaben, des klinischen Befundes und der diagnostischen Maßnahmen zu achten. Die Primärdiagnostik komplexer chronisch-entzündlicher Erkrankungen wie Psoriasis und Neurodermitis wird nicht empfohlen. In Tab. 1 werden die Empfehlungen kursorisch zusammengefasst.

SAF und RT sind als gleichwertig zu bewerten. Mit der Veröffentlichung der Leitlinie besteht erstmalig auch eine rechtliche Stütze für den Einsatz der Teledermatologie. Zurzeit bestehen jedoch zahlreiche Limitationen, die durch fehlende Evidenz für den Einsatz teledermatologischer Verfahren für einzelne Diagnosen entstehen.

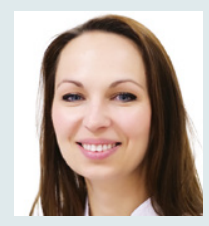

Dr. Natalia Kirsten, Ober- und Prüfärztin für Dermatologie und Venerologie CeDeF - Dermatologische Forschung und Ambulanzen, Institut für Versorgungsforschung in der Dermatologie und bei Pflegeberufen, Universitätsklinikum Hamburg-Eppendorf, Hamburg, n.kirsten@uke.de 


\section{Leitlinie Teledermatologie Die Herausforderungen werden komplexer}

Die neue Leitlinie Teledermatologie trägt zu einer verbesserten Versorgungsstruktur und Qualität bei. Allerdings wächst das Segment derzeit rapide, zahlreiche Angebote und Apps strömen auf den Markt, die Möglichkeiten der Teledermatologie werden ständig erweitert. Wo sollte die Leitlinie im nächsten Update direkt nachlegen? Ein Kommentarvon Dr. Estefania Lang, Mitgründerin dermedi-login $\mathrm{GmbH}$ und der Dermanostic GmbH.

Leitlinien bilden einen wichtigen Orientierungspunkt in unserem klinischen Alltag und spielen eine zunehmend größere Rolle in der Wahl medizinischer Diagnose- und Behandlungspfade, so auch in der Dermatologie [1, 2]. Aufgrund der in den zurückliegenden Jahren sehr positiven und vielversprechenden Entwicklungen auf dem Gebiet der Telemedizin [3] ist es nicht überraschend, dass kürzlich unter der Registernummer 013-097 nun auch eine S2k-Leitlinie der Arbeitsgemeinschaft der Wissenschaftlichen Medizinischen Fachgesellschaften (AWMF) zur Teledermatologie online veröffentlicht wurde (https://www.awmf.org/uploads/tx_szleitlinien/013-097I_S2k_ Teledermatologie_2021-03.pdf).

Es ist unbestritten, dass die Erstellung einer neuen Leitlinie die Autoren vor eine enorme Aufgabe und Herausforderung stellt. Um eine qualitativ und systematisch möglichst hochwertige Leitlinie zu verfassen, bedarf es zahlreicher Stunden in konstituierenden Sitzungen, umfangreicher Literaturrecherchen, Evidenzanalysen und nicht zuletzt einer Konsensfindung [4]. Darüber hinaus gilt es, die Unabhängigkeit von externen Finanzquellen und diversen «Interessengruppen» zu gewährleisten [5]. Der Arzt als Anwender hingegen steht vor dem Dilemma, sich oftmals mit verschiedenen Leitlinien nationaler und internationaler Fachgesellschaften zu ein und demselben Thema/Krankheitsbild konfrontiert zu sehen [4]. Hinzu kommt die Tatsache, dass in der heutigen Zeit Patienten und Patientenverbände über das Internet ungehindert Zugang zu den online veröffentlichten Leitlinien haben, und es somit immer wieder zu Diskussionen kommt, in denen der Arzt ein Abweichen von den Empfehlungen einer Leitlinie erklären muss. Hierbei wird des Öfteren außer Acht gelassen, dass Leitlinien lediglich Entscheidungshilfen darstellen und rechtlich nicht bindend sind [6].

\section{Leitlinienvorbereitung und -erstellung}

Ungeachtet der klaren Vorgaben zur Leitlinienvorbereitung seitens der AWMF sind die ersten Schritte bei der Erstellung einer neuen Leitlinie häufig die schwersten [7]. Ein wesentlicher Aspekt hierbei ist unter anderem die Auswahl und Anzahl der Autoren, die einerseits ausreichend stark und fachlich kompetent erscheinen sollte, um Leitlinien zum ausgewählten Thema zu entwickeln, andererseits aber auch klein genug, um ein Leitlinienprojekt effizient voranzutreiben [4].

Gegenwärtig gibt es mehrere und in ihrer Zahl zunehmende deutschsprachige Anbieter für die teledermatologische Konsultation über sogenannte «Apps», der Kurzform des englischen Wortes «application» (im Deutschen «Applikation») (Tab. 1). Hinsichtlich der Autorenzusammenstellung der Leitlinie Teledermatologie ist positiv hervorzuheben, dass nicht nur Hautärzte/innen aus Deutschland an deren Erstellung beteiligt waren, sondern auch Kollegen/ innen aus Österreich und der Schweiz. Bei genauer Betrachtung fällt auf, dass die Autoren, sofern sie im Rahmen der Teledermatologie als Kooperationspartner oder in anderer verantwortlicher Form mit einer teledermatologischen Konsultationsplattform assoziiert sind, vornehmlich zweien dieser Anbieter zugehören, während Hautfachärzte anderer Anbieter im Autorengremium der Leitlinie nicht vertreten sind, obwohl sie sich in den letzten Jahren wiederholt hinsichtlich der Thematik wissenschaftlich-publikatorisch sowie durch von der Allgemeinheit bzw. bundeslandspezifischen Initiativen unterstützte und/oder ausgezeichnete Projekte ausgewiesen haben $[8,9]$. Daher sollte gerade in einem so neuen und dynamischen Themenfeld aktuell und bei einer zukünftigen Fassung dieser Leitlinie die Autorenzusammenstellung besondere Beachtung finden - dies insbesondere unter dem Gesichtspunkt, von der Expertise möglichst aller auf diesem Gebiet kundigen Kollegen/innen profitieren zu können.

\section{Klassifikation der Leitlinie}

Die Festlegung der angestrebten Stufenklassifikation der zu erstellenden Leitlinie ist einer der entscheidenden Punkte in der Leitlinienvorbereitung und -erstellung. An diesem Punkt ist es wichtig, zwischen dem verständlichen Anspruch, eine qualitativ und systematisch möglichst hochwertige Leitlinie - im Idealfall eine evidenz- und konsensbasierte S3-klassizierte Leitlinie - zu erstellen, und den Ressourcen der Autorengruppe und der zum entsprechenden Thema vorhandenen Literatur abzuwägen [4].

Die hier vorliegende Leitlinie Teledermatologie ist als S2k klassifiziert, die weitestgehend auf Konsensfindung basiert. Bei der Konsensfindung sind insbesondere auch Vertreter anderer Fachgesellschaften frühzeitig zu beteiligen. Diesbezüglich wird in Abschnitt 6.2 der Leitlinie ausgeführt, dass die Deutsche Gesellschaft für Allgemeinmedizin und Familienmedizin (DEGAM) angefragt worden sei, diese aber aus Ressourcengründen keine verantwortliche Ansprechperson hätte benennen können. Im Widerspruch hierzu ist jedoch bereits den vorangehenden Angaben in Abschnitt 4 (Limitationen der Leitlinie) zu entnehmen, dass auf die Einbeziehung anderer Fachdisziplinen verzichtet wurde, da die teledermatologische Versorgung in anderen Fächern zum Teil noch wenig etabliert sei. Es muss somit festgehalten werden, dass dies eine wahrhaftige und nicht von der Hand zu weisende Limitation der vorgestellten Leitlinie darstellt. Auch stellt sich darüber hinaus die Frage, ob es nicht vielmehr von Belang wäre, wie sehr die allgemeine telemedizinische Versorgung in anderen Fachrichtungen entwickelt ist, als dass hier die teledermatologische Versorgung als Vergleich herangezogen wird. Schließlich könnten jegliche Erfahrung und sämtlicher Kenntnisgewinn anderer Fachdisziplinen auf dem Gebiet der Telemedizin auch für die Dermatologie hilfreich und von Nutzen sein und somit die Qualität der Leitlinie steigern. 
Gerade bezüglich der in der Leitlinie näher besprochenen Psoriasis wäre die Einbeziehung anderer Fachdisziplinen wie der Rheumatologie oder der Inneren Medizin sicherlich von Nutzen gewesen. Denn auch wenn es beispielsweise in der Rheumatologie gegenwärtig (noch) weniger Anbieter telerheumatologischer «Apps» geben mag als in der Dermatologie [10], so ist es nicht von der Hand zu weisen, dass es sie gibt und diese auch bereits wissenschaftlich evaluiert wurden (Tab. 2) [11].

Da eine wesentliche Manifestationsform und Komplikation der Psoriasis, die Psoriasis-Arthritis, bei etwa 20-30\% der Patienten mit Psoriasis vulgaris auftritt, wären in diesem Zusammenhang insbesondere die Apps «RheumaBuddy» und «RheumaVOR» hervorzuheben. «RheumaBuddy» bietet Menschen mit einer rheumatoiden Arthritis oder juvenilen idiopathischen Arthritis die Möglichkeit, ihre Symptome in einem digitalen Tagebuch zu verfolgen. Diese App wurde in enger Zusammenarbeit von Rheumatologen und Patienten entwickelt, um die individuelle Krankheitsentwicklung zwischen aufeinanderfolgenden Arztbesuchen zu dokumentieren. «Rheuma-VOR» ist eine App des Netzwerks für die «Verbesserung der rheumatologischen Versorgungsqualität durch koordinierte Kooperation», ein durch die Bundesländer Rheinland-Pfalz, Saarland und Niedersachen gefördertes Projekt. Ziel ist ein Rheuma-Schnell-«Screening», um bei den ersten Anzeichen einer rheumatoiden Arthritis, Spondylarthritis oder Psoriasis-Arthritis rasch zu einer Diagnose zu gelangen [12]. Zudem steht die Psoriasis vulgaris in engem Zusammenhang mit dem metabolischen Syndrom und verschiedenen seiner Komponenten (Adipositas, erhöhte Plasmaglukose, Erhöhung von Cholesterin und Triglyzeriden, Hypertonie) - eine Domäne nicht nur auf die Psoriasis spezialisierter dermatologischer Zentren, sondern auch der Inneren Medizin [13, 14]. Auch wenn bezüglich der Inhalte und Anwendungsgebiete eine 1:1-Übertragung auf die Dermatologie weder möglich noch sinnvoll erscheint, so gibt es doch bereits wesentliche Evidenz, die auch bei der Einstufung dermatologischer Fragestellungen hilfreich sein könnte.
Tab 1.: Übersicht zu deutschsprachigen Teledermatologie-Apps (ohne Anspruch auf Vollständigkeit)

\begin{tabular}{|c|c|c|c|c|}
\hline App & Zielgruppe & Zweck & Preis & $\begin{array}{l}\text { Betriebs- } \\
\text { system }\end{array}$ \\
\hline $\begin{array}{l}\text { Dermanostic - } \\
\text { Hautarzt per App }\end{array}$ & Patienten & $\begin{array}{l}\text { Diagnosefindung/ } \\
\text { Therapieeinleitung }\end{array}$ & kostenlos & $\begin{array}{l}\text { iOS/Android, } \\
\text { WebApp }\end{array}$ \\
\hline derma2go & Patienten & $\begin{array}{l}\text { Diagnosefindung/ } \\
\text { Therapieeinleitung }\end{array}$ & kostenlos & WebApp \\
\hline First Derm & Patienten & $\begin{array}{l}\text { Diagnosefindung/ } \\
\text { Therapieeinleitung }\end{array}$ & kostenlos & $\begin{array}{l}\text { iOS/Android, } \\
\text { WebApp }\end{array}$ \\
\hline SkinDoc24 - Hautarzt & Patienten & $\begin{array}{l}\text { Diagnosefindung/ } \\
\text { Therapieeinleitung }\end{array}$ & kostenlos & $\begin{array}{l}\text { iOS/Android, } \\
\text { WebApp }\end{array}$ \\
\hline OnlineDoctor & Patienten & $\begin{array}{l}\text { Diagnosefindung/ } \\
\text { Therapieeinleitung }\end{array}$ & kostenlos & WebApp \\
\hline $\begin{array}{l}\text { Online Hautarzt - } \\
\text { AppDoc }\end{array}$ & Patienten & $\begin{array}{l}\text { Diagnosefindung/ } \\
\text { Therapieeinleitung }\end{array}$ & kostenlos & $\begin{array}{l}\text { iOS/Android, } \\
\text { WebApp }\end{array}$ \\
\hline
\end{tabular}

Tab 2.: Übersicht zu Apps in der Rheumatologie (ohne Anspruch auf Vollständigkeit)

\begin{tabular}{|c|c|c|c|c|}
\hline App & Zielgruppe & Zweck & Preis & $\begin{array}{l}\text { Betriebs- } \\
\text { system }\end{array}$ \\
\hline ABATON & $\begin{array}{l}\text { Patienten/ } \\
\text { Ärzte }\end{array}$ & $\begin{array}{l}\text { Verlaufsbeob- } \\
\text { achtung }\end{array}$ & kostenlos & iOS/Android \\
\hline Kitchen Stories & Patienten & $\begin{array}{l}\text { gesunde } \\
\text { Ernährung }\end{array}$ & kostenlos & iOS/Android \\
\hline MyTherapy & Patienten & $\begin{array}{l}\text { Therapieunterstüt- } \\
\text { zung/ Gesund- } \\
\text { heitstagebuch }\end{array}$ & kostenlos & iOS/Android \\
\hline Relax Melodies & Patienten & $\begin{array}{l}\text { Achtsamkeit/ } \\
\text { Entspannung }\end{array}$ & kostenlos & iOS/Android \\
\hline RhePort & $\begin{array}{l}\text { Patienten/ } \\
\text { Ärzte }\end{array}$ & $\begin{array}{l}\text { Patienten- } \\
\text { edukation/ } \\
\text { Terminvergabe }\end{array}$ & kostenlos & WebApp \\
\hline Rheuma Auszeit & Patienten & $\begin{array}{l}\text { Entspannung/ } \\
\text { Schmerzbewälti- } \\
\text { gung }\end{array}$ & kostenlos & iOS/Android \\
\hline RheumaBuddy & Patienten & $\begin{array}{l}\text { Therapieunter- } \\
\text { stützung/ Gesund- } \\
\text { heitstagebuch }\end{array}$ & kostenlos & iOS/Android \\
\hline RheumaGuide & Ärzte & Nachschlagewerk & kostenlos & iOS/Android \\
\hline $\begin{array}{l}\text { Rheuma Schweiz Edu- } \\
\text { cation }\end{array}$ & Ärzte & Nachschlagewerk & kostenlos & iOS/Android \\
\hline Rheuma-VOR & Ärzte & Diagnosefindung & kostenlos & iOS/Android \\
\hline Runtasty & Patienten & $\begin{array}{l}\text { gesunde } \\
\text { Ernährung }\end{array}$ & kostenlos & iOS/Android \\
\hline
\end{tabular}




\section{Thematische und inhaltliche Empfehlungen der Leitlinie}

Die Leitlinie behandelt hinsichtlich ihrer konkreten Empfehlungen

5 Themen oder Krankheitsbilder:

1. Psoriasis

2. Atopische Dermatitis und andere Ekzemerkrankungen

3. Hautkrebs

4. Chronische und akute Wunden

5. Weitere dermatologische Anwendungsbereiche

\section{Psoriasis}

Bezüglich der Psoriasis lautet die erste Empfehlung in Abschnitt 3.1 der Leitlinie, dass eine Erstdiagnostik der Erkrankung allein auf der Basis eines teledermatologischen Befundes nicht erfolgen solle, wofür ein starker Konsens (100\%) erreicht wurde. Wie diese Konsensfindung mit den Ergebnissen mehrerer randomisierter Studien vereinbar ist, die zur Diagnostik und Therapie der Psoriasis keinen Unterschied zwischen der teledermatologischen und der Vor-OrtKonsultation zeigen konnten, bleibt offen $[15,16]$. Ein ebenso starker Konsens konnte für die Empfehlung erzielt werden, dass die teledermatologische Bestimmung des wichtigen Psoriasis Area and Severity Index (PASI) aufgrund der fehlenden Möglichkeit zur Beurteilung der Induration nicht erfolgen könne. Diese Einschätzung ist jedoch sicherlich kontrovers zu diskutieren, wenn nur zwei Tabellenzeilen zuvor mit ebenso starkem Konsens empfohlen wird, dass die Psoriasis bei erfolgter Diagnosestellung durch eine Präsenzuntersuchung grundsätzlich mit einer Store-and-Forward (SAF)- oder Realtime (RT)-Technologie im Verlaufe begutachtet werden könne. Somit stellt sich konsequenterweise die Frage, wie denn der PASI im Verlauf erhoben und somit zur Kontrolle des Behandlungserfolges herangezogen werden könnte, wenn dies im Rahmen der Erstdiagnostik nicht möglich sein soll.

\section{Atopische Dermatitis und andere Ekzemerkrankungen}

Obwohl in der Überschrift zu Abschnitt 3.2 thematisiert, wird in dieser Sektion nicht auf andere Ekzemerkrankungen eingegangen, sondern die detaillierten Empfehlungen beziehen sich ausschließlich auf das atopische Ekzem. Auch diesbezüglich lautet die erste Empfehlung, dass eine Erstdiagnostik der Erkrankung allein auf der Basis eines teledermatologischen Befundes nicht erfolgen solle, wofür - wie bei der Psoriasis - ein starker Konsens (100\%) erzielt wurde. Ein ebenso starker Konsens konnte auch für die in der sechsten Zeile der Tabelle genannte Empfehlung erreicht werden, dass das «Ödem» teledermatologisch nicht hinreichend beurteilt werden könne und somit eine Erhebung des Severity Scoring of Atopic Dermatitis (SCORAD)-Wertes und des Eczema Area and Severity Index (EASI) nicht sachgerecht möglich sei. Abgesehen davon, dass ein «Ödem» im strengen Sinne nicht zu den Kriterien der beiden zuvor genannten «Scores» zählt, überrascht es dann jedoch ebenso wie bei der Psoriasis, wenn nur drei Tabellenzeilen zuvor mit ebenso starkem Konsens empfohlen wird, dass die atopische Dermatitis bei erfolgter Diagnosestellung durch eine Präsenzuntersuchung grundsätzlich mit einer SAFoder RT-Technologie im Verlauf begutachtet werden könne. Dies wirft abermals die Frage auf, wie denn der SCORAD-Wert und der
EASI im Verlauf erhoben und somit zur Kontrolle des Behandlungserfolges herangezogen werden könnten, wenn dies im Rahmen der Erstdiagnostik nicht möglich ist.

\section{Fazit}

Die Initiative zur Erstellung einer Leitlinie zum Thema Teledermatologie ist unzweifelhaft äußerst unterstützungswürdig und insbesondere vor dem Hintergrund der teilweise wochenlangen Wartezeiten auf einen Termin beim Hautfacharzt sowie der seit Dezember 2019 herrschenden COVID-19-Pandemie sehr wichtig, da die Telemedizin und vor allem die Teledermatologie seit ihrer Implementierung eine unbestreitbar wertvolle Ergänzung zur klassischen persönlichen (Erst-)Konsultation eines Hautarztes durch Menschen mit einer Hauterkrankung darstellt $[2,8,9,17]$.

Dennoch erscheint es uns wünschenswert, dass die hier angesprochenen Punkte in ihrer Gesamtheit zu Anpassungen und Verbesserungen zukünftiger Aktualisierungen der Leitlinie führen - ebenso wie zu reger Diskussion. Ziel muss es sein, unsere Patienten bestmöglich zu beraten, zu begleiten und zu behandeln. Die Frage, welche teledermatologischen Instrumente und Verfahren bei einem Patienten zu einem bestimmten Zeitpunkt zum Einsatz kommen könnten und sollten, bedarf sicherlich weiterer Studien. Dort aber, wo bereits Expertise und Evidenz vorhanden sind, sollten diese auch beachtet, genutzt und herangezogen werden.

\section{Disclosure Statement}

Die Autoren erklären, dass sie sich bei der Erstellung des Beitrages nicht von wirtschaftlichen Interessen leiten ließen. Sie legen die folgenden potenziellen Interessenkonflikte offen:

E.L. ist Geschäftsführerin und Mitgründerin der medi-login GmbH sowie Mitgründerin und medizinische Leitung der Dermanostic GmbH. Sie erhielt Beratungs- und Vortragshonorare von Galderma GmbH, Mylan GmbH, Viatris GmbH, Novartis Pharma, Euroimmun AG, Heine Optotechnik GmbH, Beiersdorf AG, LÓREAL, Roche Pharma.

P.S. erhielt Beratungs- und Vortragshonorare von AXIOM Health, Amgen, AbbVie, Biogen, Bristol-Myers Squibb, Celgene, Chugai Pharma Marketing Ltd./Chugai Europe, Deutscher Psoriasis-Bund, Gilead Sciences, Hexal Pharma, Janssen-Cilag, Johnson \& Johnson, Lilly, Mediri GmbH, Novartis Pharma, Onkowissen GmbH, Pfizer, Roche Pharma, Rheumazentrum Rhein-Ruhr, Sanofi-Genzyme, Spirit Medical Communication, Swedish Orphan Biovitrum und UCB Pharma; und Forschungsförderung von Deutsche Forschungsgesellschaft (DFG), Bundesministerium für Bildung und Forschung (BMBF), European Union (EU), Amgen, AbbVie, BMS, Celgene, Chugai, Deutscher Psoriasis-Bund, Janssen-Cilag, Lilly, Novartis, Roche und UCB Pharma.

\section{Literatur}

1. Encke A, Kopp I, Selbmann H-K: Allgemein- und Viszeralchirurgie up2date 2009;3:257-267.

2. Lehmann L, Nittas V, et al.: Kompass Dermatol 2020;8:160-163.

3. Elsner P, Bauer A, Diepgen TL, et al.: J Dtsch Dermatol Ges 2018;16:969-974.

4. Nach T: Uro-News 2014;18:28-31.

5. Rosumeck S, Sporbeck B, Rzany B, Nast A: J Dtsch Dermatol Ges 2011;9:297-304.

6. Harneit P: MKG-Chirurg 2009;2:107-112.

7. Ständige Kommission «Leitlinien» der AWMF: AWMF-Regelwerk «Leitlinien», ed 1. www.awmf.org/leitlinien/awmf-regelwerk.html, 2012 (Zugriff am 25.04.2021).

8. Sondermann W, von Kalle C, Utikal JS, Schadendorf D, Esser S, Durani B, et al.: Hautarzt 2020;71:887-897.

9. Brinker TJ, Hekler A, von Kalle C, Schadendorf D, Esser S, Berking C, et al.: J Med Internet Res 2018;20:e11871.

10. Lang E, Martin A, Frank J: arthritis + rheuma 2021, in press.

11. Grahammer M: arthritis + rheuma 2021, in press.

12. Schwarting A, Dreher M, Assmann G, Witte T, Hoeper K, Schmidt RE: Z Rheumatol 2019;78:743-752.

13. Gerdes S, Mrowietz U, Boehncke WH: Hautarzt 2016;67:438-444. 
14. Peralta C, Hamid P, Batool H, Al Achkar Z, Maximus P: Cureus 2019;11:e6369.

15. Armstrong AW, Chambers CJ, Maverakis E, Cheng MY, et al.: JAMA Netw Open 2018;1:e183062.

16. Beer J, Hadeler E, Calume A, Gitlow H, Nouri K: Arch Dermatol Res 2021;313:11-15.

17. Elsner P: J Dtsch Dermatol Ges 2020;18:841-845.

\section{Danksagung}

E.L. wurde unter anderem ausgezeichnet durch den ersten Platz bei «ERCIS Lauch Pad 2020» des European Research Center for Information Systems der Westfälischen Wilhelms-Universität Münster, den dritten Preis bei «Zheng-He-Pokal Jugendinnovations- und Unternehmerpreis 2020» der Chinesisch-Deutschen Akademischen Gesellschaft e.V. und den zweiten Platz bei «BioRiver Boost 2020» des Life Science im Rheinland e.V. sowie als Preisträgerin im Unternehmenswettbewerb «NRW Wirtschaft im Wandel 2020» durch Land der Ideen Management GmbH.

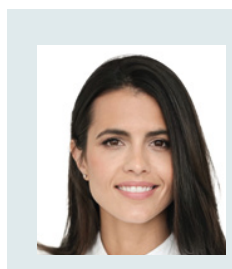

Estefanía Lang (im Bild) ${ }^{a, b}$, Philipp Sewerinc; aDermanostic Online-Praxis für Dermatologie, Düsseldorf, Deutschland; 'b Institut für Gesundheit und Soziales, FOM Hochschule für Oekonomie \& Management GmbH, Essen, Deutschland; 'Poliklinik, Funktionsbereich und Hiller Forschungszentrum für Rheumatologie, Heinrich-Heine-Universität Düsseldorf, Düsseldorf, Deutschland Kontaktadresse:

Dr. med. Estefanía Lang, e.lang@dermanostic.com

\section{Digitalisierung in der Onkologie Chancen und Nutzen in der klinischen Praxis}

Digitale Tools sind für die Ärzteschaft wie für die Pflegenden vielfach noch ungewohnt. Ihr Nutzen erschließt sich nicht immer auf den ersten Blick. Das betrifft nicht nur die Dermatologie, ein Blick über den Tellerrand lohnt sich. Dr. Friedrich Overkamp und Emre Basar blicken für uns auf die Entwicklungen, die in der Onkologie die klinische Praxis in hohem Maße verändern werden.

Ein Beispiel für diese Entwicklungen sind die sogenannten Wearables. Unter Wearables (wireless sensors) werden drahtlose Messgeräte verstanden, die in der Lage sind, diverse Parameter zu messen. So gibt es die Idee, den Zuckerspiegel der Tränenflüssigkeit über einen smarten Sensor in einer Kontaktlinse zu messen. Der in der Tränenflüssigkeit gemessene Wert soll an eine App gemeldet werden, die eine Korrelation mit dem Blutzuckerspiegel herstellt und eine Insulinpumpe steuert. Auch wenn noch nicht feststeht, ob der Prototyp (die «Google Linse») weiterentwickelt wird, zeigt die Idee sehr eindrücklich, in welche Richtung sich die digitale Forschung entwickelt. Neben Google treibt auch Apple eine technologisch höchst anspruchsvolle Entwicklung voran, um den Blutzuckerspiegel nicht-invasiv via Smartwatch zu messen. Mutmaßlich wird bereits die nächste Generation der Apple Watch (iWatch 7) mit einem Infrarot-basierten Blutanalyse-Sensor ausgestattet sein, um den Glucosespiegel zu erfassen.

Drahtlose und für onkologische Bedürfnisse akzeptable EKG Ableitungen können z.B. mit den Devices von «Alivecor» (www.alivecor. com) oder der «Apple Watch 4» durchgeführt werden, die bereits seit September 2018 von der FDA in den USA und auch in mehreren europäischen Ländern zugelassen ist. Damit lässt sich z.B. Vorhofflimmern diagnostizieren oder ausschließen [1]. Neuere Smartphones tragen auf ihrer Rückseite einen Sensor, der bei Berührung eines Fingers in der Lage ist, Blutdruck und Puls ziemlich exakt über kleinste Arteriolen der Fingerkuppe zu messen [2]. Auch ein Blutbildanalysegerät für zu Hause existiert schon (www.athelas.com), das in der Lage ist, in kürzester Zeit ein Differenzialblutbild zu bestimmen: Ein kleiner Bluttropfen aus der Fingerkuppe (ähnlich wie bei der Blutzuckerbestimmung) wird auf ein Spezialpapier aufgetragen und dieses in ein Messgerät geschoben, das kleiner ist als ein digitales Kommunikationsgerät wie «Alexa».

Auch smarte Tabletten sind bereits auf dem Markt, die melden, wenn sie im Magen angekommen sind, quasi als digitale AdhärenzKontrolle. Ein Beispiel ist Aripiprazol (Abilify MyCite(R)), ein Psychopharmakon, das sehr regelmäßig eingenommen werden muss. In die Tablette wurde ein smarter Faden integriert, der bei Kontakt mit Magensäure ein schwaches elektromagnetisches Signal aussendet, das von einer App im Smartphone erkannt wird [3, 4]. Empfängt die App das Signal zu einem erwarteten Zeitpunkt, gilt die Tablette als korrekt eingenommen. Bleibt das Signal aber aus, dann schlägt die App «Alarm», indem sie z.B. den Wecker aktiviert oder automatisch Kontaktpersonen anruft. Auch in der onkologischen Pharmazie wird die Entwicklung von Oralia mit einer smarten Einnahmekontrolle diskutiert.

\section{Telemedizin}

Aktuell setzen die gesetzlichen Krankenversicherungen auf den schnellen Ausbau von Video-Sprechstunden. Man erwartet, dass in 5 Jahren jede 5. Behandlung per Videosprechstunde erfolgt (Vorstand GKV Spitzenverband, Januar 2020). Fernbehandlung (Telemedizin) kann insbesondere in ländlichen Regionen hilfreich werden. Aber auch unabhängig von der Region sind viele Nutzungsbereiche der Telemedizin denkbar, bei denen nicht zwingend der Besuch in einer Praxis oder Klinikambulanz notwendig ist. Als ein weiterer sinnvoller Anwendungsbereich für die Telemedizin dürfte sich die Behandlung von Patienten mit seltenen Erkrankungen herauskristallisieren, denn gerade für diese Patientengruppe ist der Weg zum nächstgelegenen Exzellenz-Zentrum oft weit und beschwerlich.

Im hausärztlichen Bereich dürften sich Videosprechstunden schnell durchsetzen, z.B. bei harmlosen Infekten, für die Einholung einer AU oder um die Notwendigkeit einer Überweisung zu klären. In der Onkologie ist die Kontrolle von kutanen Nebenwirkungen einer Therapie (z.B. Hand-Fuß-Syndrom oder immunvermittelte Dermatitis) ein Beispiel für die sinnvolle Nutzung telemedizinischer Abklärung, da die meisten Hautveränderungen über ein mit dem Smartphone angefertigtes Foto genauso gut diagnostiziert werden können wie bei einer direkten Konsultation. Für Ärztinnen und Ärzte
160

Kompass Dermatol 2021;9:152-162 DOI: $10.1159 / 000516815$ 
ebenso wie für Pflegende in der Onkologie werden Videosprechstunden in der Zukunft eine Bereicherung sein, da damit die Versorgung der Patientinnen und Patienten zeitnäher und effizienter durchgeführt werden kann.

Ein weiterer Bereich mit rasch zunehmender Nutzung telemedizinischer Möglichkeiten dürfte die Diskussion radiologischer Bilder im Kollegenkreis sein, z.B. im Rahmen von online durchgeführten Tumor-Boards. Zudem werden radiologische Bilder künftig auch direkt in einer elektronischen Patientenakte zur Verfügung stehen. Erste Apps, die von Krankenkassen herausgegeben werden (z.B. «Vivy») lassen bereits heute die Speicherung von Bildern und Befunden zu. Im Bereich der Robotor-Chirurgie in urologischer oder gynäkologischer Onkologie werden schon routinemäßig telemedizinische Konsultationen intraoperativ bei speziellen Fragestellungen genutzt.

Zweifelsohne hat die Coronavirus-Pandemie die Akzeptanz für telemedizinische Anwendungen beschleunigt und führenden europäischen Telemedizin-Anbietern wie Kry und Babylon Health einen enormen Wachstumsschub beschert.

\section{Big Data und Künstliche Intelligenz}

Big Data - die Sammlung, Analyse und Nutzung immenser Datenmengen - wird die Onkologie sehr stark voranbringen. Die Entwicklung künstlicher Intelligenz wird insbesondere Diagnostikfächer wie Radiologie, Pathologie und Dermatologie in hohem Maße verändern. Es zeichnet sich ab, dass Algorithmen, lernende Computerprogramme, in Zukunft schneller und präziser diagnostizieren können als das menschliche Gehirn. Für das Potential künstlicher Intelligenz werden nahezu monatlich neue Belege publiziert. So wurde z.B. gezeigt, dass Algorithmen Mikrometastasen von Mammakarzinomen in axillären Lymphknoten mindestens genauso gut erkennen können wie Pathologen [5]. Ebenso wurde dokumentiert, dass Künstliche Intelligenz in der Lage ist, Areale mit MSI high in Gewebeschnitten zu erkennen [6] oder dass sie in der Lage ist, verdächtige Areale im Prostata-MRT ähnlich gut zu erkennen wie Radiologen [7]. Es ist absehbar, dass die Befunde von Schnittbilduntersuchungen wie CT oder MRT künftig mehr und mehr von Algorithmen erstellt werden [8]. Zebra Medical Vision als einer der Vorreiter in diesem Feld hat bereits mehrere von der FDA genehmigte Produkte auf den Markt gebracht, die basierend auf künstlicher Intelligenz Radiologen bei der Diagnose verschiedener Erkrankungen (wie Osteoporose, Brustkrebs) unterstützen.

Ebenso dürfte das Hautkrebsscreening schon bald von SmartphoneKameras und angeschlossenen Algorithmen bewerkstelligt werden können: «Lesions learnt» [9]. In rascher Entwicklung befinden sich auch lernende App-Programme, die aus eingegebenen anamnestischen Angaben mit zunehmend höherer Treffsicherheit Verdachtsdiagnosen stellen (z.B. www.ada.com). Die Nutzung der über solche Tools erzeugten Daten ist jedoch derzeit Gegenstand datenschutzrechtlicher und medizinethischer Diskussionen. Wenn diese grundlegenden Fragen geklärt sind, dürften in Zukunft Patienten z.B. zu Hause oder im Wartezimmer gebeten werden, vor dem Arztkontakt ihre Symptome in eine App einzugeben, was eine Vorsortierung der Probleme und damit eine gezieltere und effizientere Behandlung ermöglicht.
Mit «Big Data» sind aber nicht nur die beschriebenen lernenden Computerprogramme gemeint. Die ungeheuren Mengen von klinischen und molekular-genetischen Daten ermöglichen eine immer präzisere Einteilung der Tumorerkrankungen in diverse Subgruppen, die eine zunehmend molekular gesteuerte und immer stärker individualisierte Therapie nach sich ziehen werden. Letztlich dürfte in der Verknüpfung von molekularem Wissen und lernenden Algorithmen die Zukunft der onkologischen Diagnostik und Therapiesteuerung liegen.

\section{Digitales Follow Up}

Ein weiteres Feld, auf dem sich digitale Ansätze für die Onkologie abzeichnen, ist die digitale Verlaufskontrolle unter einer Tumortherapie. Die Sinnhaftigkeit einer Implementierung von elektronisch erhobenen Daten zum patient reported outcome (ePRO) wurde durch zahlreiche Arbeitsgruppen publiziert [10]. Durch die Arbeiten von Denis et al. 2016 [11] und Basch et al. 2017 [12] ist darüber hinaus klar, dass digitale Tools einen hohen Stellenwert beim so genannten Follow Up entfalten können. In der von Basch et al. durchgeführten Studie wurde deutlich, dass eine regelmäßige präzise online Abfrage von Symptomen und Nebenwirkungen für die Patienten von Vorteil sein kann: In einer Studie mit ca. 1000 Patienten mit verschiedenen Krebserkrankungen und unterschiedlichen Therapien erhielt die eine Hälfte ein «klassisches follow up» mit einem fest terminierten Besuch in der onkologischen Einrichtung alle 8-12 Wochen für die üblichen Kontrolluntersuchungen. Die andere Hälfte der Studienteilnehmer erhielt keinen festen Termin, sondern nur einmal pro Woche eine E-Mail mit einem Link zu einer Plattform, auf der man sich einloggen musste. Auf dieser Plattform wurden den Patientinnen und Patienten jede Woche 12 Fragen gestellt, die zu ihrer jeweiligen Therapie passten (z.B. Fragen nach typischen Nebenwirkungen oder nach tumorbedingten Symptomen). Die Antworten wurden von onkologischen Pflegern und Ärzten gecheckt. Wenn alles im grünen Bereich war, wurde nichts unternommen und der Patient bekam eine Woche später wieder 12 Fragen gestellt. Sobald jedoch eine Antwort zu verzeichnen war, die auf eine Nebenwirkung, eine Komplikation oder einen Progress hinzudeuten schien, wurden die betreffenden Patienten sofort einbestellt und es wurde interveniert. Interessanterweise hatte die Gruppe mit der digitalen Überwachung nur Vorteile gegenüber der Gruppe mit der klassischen, fest terminierten ärztlichen Kontrolle: Ihre Lebensqualität war besser, es waren seltener Notfallinterventionen nötig, und es war sogar ein Überlebensvorteil der digital überwachten Patientinnen und Patienten nachweisbar. Die Überprüfung und Umsetzung dieser wissenschaftlichen Erkenntnisse erfolgt in Deutschland z.Z. mit verschiedenen Tools, u.a. mit der «Cankado» App, einem digitalen Tagebuch für Krebspatienten, die als Medizinprodukt für die Erfassung von Symptomen, Adhärenz und Lebensqualität sowie zum Therapiemanagement genutzt werden kann und in verschiedenen onkologischen Studien eingesetzt wird [13]. Weitere, ähnliche Tools sind «Kaiku Health» oder «Babylon Health».

Die Beispiele zeigen, welche faszinierenden digitalen Chancen sich für die Onkologie abzeichnen. Unabdingbar ist, dass die neuen digitalen Tools Zertifizierungsprozesse durchlaufen und in klinischen 
Studien validiert werden, d.h. ihre Nützlichkeit und Sicherheit unter Beweis stellen. Eine erste onkologische «App auf Rezept» dürfte mit hoher Wahrscheinlichkeit aus diesem Bereich stammen.

\section{Digitale Wissensvermittlung und Wissensmanagement}

Besonders spürbar ist die Digitalisierung bereits jetzt im Bereich der Wissensvermittlung und des Wissensmanagements. Eine digitale Verfügbarkeit des Wissens ist die wichtigste Voraussetzung für eine zeitgemäße Onkologie. Mit der Entwicklung von Smartphones und Tablets hat sich seit etwa einem Jahrzehnt ein beträchtlicher Teil der Internetnutzung kontinuierlich von der klassischen Website auf Apps verlagert.

Hauptunterschied zwischen kommerziellen und nicht-kommerziellen Anbietern ist, dass die Apps von kommerziellen Anbietern i.d.R. aufwändiger programmiert und verlinkt sind. Obwohl industriell unterstützt, haben auch die kommerziellen Anbieter den Anspruch akademische Tools zu sein, produktneutral zu informieren, den aktuellen Stand des Wissens in modernem App-Design abzubilden sowie relevante nationale und internationale Leitlinien zu berücksichtigen.

Verschiedene Angebote sind z.Z. in Entwicklung, Beispiele sind die App «EasyOncology» oder die Apps von «onkowissen.de». «Easy Oncology» bietet eine professionelle Unterstützung bei Therapieentscheidungen für erfahrene Therapeuten, «onkowissen.de» setzt auf einen eher edukativen Ansatz. Nach dem derzeitigen Entwicklungsstand ergänzen sich die Inhalte beider Tools. Die Angebote bereiten den Stand des Wissens und die zur Verfügung stehenden diagnostischen und therapeutischen Optionen so auf, dass sie mühelos und zu jeder Zeit auf mobilen Endgeräten verfügbar sind.

Auch klassische Leitlinienportale wie «Onkopedia» oder die «S3 Leitlinien der AWMF» werden bereits in App-Form herausgegeben, so dass sie auch auf mobilen Endgeräten gelesen werden können. Entscheidend ist für alle Angebote, dass sie ständig aktuell gehalten werden. Ergebnisse wegweisender Publikationen oder Highlights von Weltkongressen sollten so zeitnah wie möglich auch in den Apps verfügbar sein. onkowissen.de hat z.B. als zusätzlichen Service Berichterstattungen von relevanten Weltkongressen auf der Partnerplattform onkowissen.tv etabliert. Die dort angeboten Kurzvideos sind auch über die Apps verfügbar, ebenso wird regelmäßig auf wegweisende Publikationen oder gesundheitspolitische Entscheidungen hingewiesen und die Nutzer werden im Falle von Neuigkeiten über entsprechende ICONs informiert.

Die Nutzung von Spracheingabesystemen analog Alexa oder Siri sowie die Integration von Chatbots für Frage-Antwort-Dialoge werden in naher Zukunft sicher auch bei der Bereitstellung des on- kologischen Wissens in Apps eine Rolle spielen.

Nutzungsanalysen zeigen, dass Apps offenbar anders genutzt werden als klassische Websites: Während letztere eher in den Abendstunden und an Wochenenden aufgerufen werden, erfolgt die Nutzung von Apps auf mobilen Endgeräten deutlich häufiger während der Sprechstunden- bzw. Visitenzeiten. Die durchschnittliche Verweildauer bei einzelnen Kapiteln ist etwas kürzer, die Zahl der Wiederkehrer dafür aber deutlich höher. Auch tagesaktuelle Ereignisse, z.B. die Zulassung eines neuen Medikamentes oder die Veröffentlichung einer Nutzenbewertung wirken sich erkennbar auf die Nutzung der Apps aus, weil an diesen Tagen verstärkt schnell auffindbare Informationen zu den jeweiligen Substanzen gesucht werden. Diese messbaren Trends sowie die Downloadzahlen signalisieren, dass offenbar ein Bedarf nach digitaler Wissensvermittlung besteht. Eine wachsende Bedeutung dürften auch in-App-Angebote für eine CME-Zertifizierung erlangen. In den Apps von onkowissen.de werden seit Ende 2019 neben den edukativen Kapiteln auch CMETools angeboten, über die man digitale Prüfungen absolvieren und CME-Punkte generieren kann. Erste Zertifizierungen einer Landesärztekammer liegen vor, so dass im Falle einer bestandenen Prüfung über die digital eingegebene EFN-Nummer entsprechende CME-Punkte automatisch gutgeschrieben werden.

\section{Disclosure Statement}

F. Overkamp ist Geschäftsführender Gesellschafter der onkowissen.de GmbH Würzburg

\section{Literatur}

1. Perez MV et al.: NEJM 2019; 381:1909-1917

2. Chandrasekhar A, et al.: Sci Transl Med. 2018 Mar 7;10(431):eaap8674.

3. Kopelowicz A et al.: Neuropsychiatr Dis Treat. 2017 Oct 19;13:2641-2651.

4. Fowler JC et al.: Neuropsychiatr Dis Treat. 2021 Feb 12;17:483-492.

5. Ehteshami Bejnordi B et al.: JAMA. 2017 Dec 12;318(22):2199-2210.

6. Kather JN et al.: Nat Med. 2019 Jul;25(7):1054-1056.

7 Belfiore MP et al.: Radiol Med. 2020 May;125(5):500-504.

8. Allen B Jr et al.: J Am Coll Radiol. 2019 Sep;16(9 Pt A):1179-1189.

9. Esteva A et al.: Nature. 2017 Feb 2;542(7639):115-118.

10. Wallwiener M et al.: Geburtshilfe Frauenheilkd. 2017 Aug;77(8):870-78.

11. Denis Fet al.: J. Clin. Oncol. 34(18 Suppl.),

12. Basch E et al.: J. Clin. Oncol. 34(6), 557-565 (2016).

13. Eggersmann, Harbeck, Schinkoethe \& Riese DOI: 10.2217/bmt-2017-0005

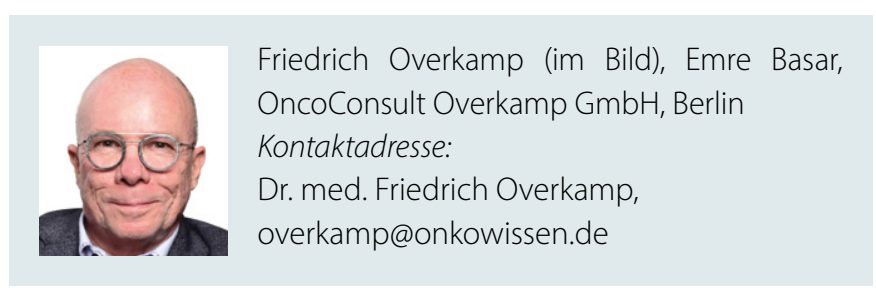

